\title{
Conférences expérimentales à l'Espace Pierre-Gilles de Gennes
}

José Bico(1) (jbico@pmmh.espci.fr), Étienne Guyon(1) (guyon@pmmh.espci.fr) et Roland Lehoucq ${ }^{(2)}$ (roland.lehoucq@cea.fr)

(1) Physique et Mécanique des Milieux Hétérogènes, ESPCI, UPMC, 7 Quai Saint Bernard, 75005 Paris

(2) Département d'astrophysique, Orme des Merisiers, Bât. 709, CEA/Saclay, 91191 Gif-sur-Yvette Cedex

Après un rappel historique sur les démonstrations publiques d'expériences scientifiques depuis les cabinets de physique du $18^{\mathrm{e}}$ siècle, cet article présente les leçons expérimentales mises en place à l'ESPCI depuis 2003, qui se sont donné l'objectif de présenter « en direct » la science d'aujourd'hui. Ces conférences, gratuites et ouvertes à tous,

qui montrent aussi bien

la manipulation d'une chaîne

d'ADN que des expériences avec des jeux, sont filmées et mises à disposition sur Canal-U pour les rendre durablement accessibles au public.

À titre d'exemple, deux encadrés décrivent des extraits d'une leçon donnée en 2017 par R. Lehoucq, sur l'utilisation de jouets (le yo-yo et le ressort slinky) pour parler de physique.
La place de l'expérimentation dans les cours de sciences physiques apparaît comme un complément indispensable des enseignements de base. Elle nous a permis, pour les plus anciens d'entre nous, de bénéficier de spectaculaires " cours de chaire " avec l'aide directe du chef de travaux et de l'assistant, des denrées rares aujourd'hui ! De façon générale, la recommandation, souvent répétée, d'associer l'expérience au savoir formel que diffusent les enseignants, n'a pas bien été suivie d'effets. C'est ce que montrent en particulier les travaux de Nicole Hulin [1], analysant des réformes successives sur plus d'un siècle, ainsi que les recommandations répétées de la Société Française de Physique (comme celles de la commission Lagarrigue-Hulin).

Le présent article rappelle d'abord schématiquement quelques étapes de cette histoire. Puis il présente les conférences expérimentales conduites à l'École supérieure de physique et de chimie industrielles de la ville de Paris (ESPCI) depuis quinze ans et héritières de leçons initiées par Faraday. Enfin, nous l'illustrons par la description et l'analyse d'une leçon récente sur des manipulations de jouets simples.

\section{Une préhistoire à partir des muséums}

Étymologiquement, musée renvoie au terme grec mouseîn, lieu de culte dédié aux Muses et à la conservation de l'ensemble des savoirs qui leur sont associés, comme celui d'Alexandrie. Bien longtemps après, c'est la découverte des nouveaux mondes et les retours des expéditions avec leurs collections qui sont des éléments essentiels dans l'apparition des cabinets de curiosité. À partir du $17^{\mathrm{e}}$ siècle, se créent également des jardins botaniques (tel que le Jardin des Plantes à Paris, initié par Guy de la Brosse), dont la triple fonction était de collectionner, classer (la taxonomie) et diffuser la connaissance. Ce seront aussi les éléments de base dans la création des muséums de science.

\section{Des cabinets de physique au Conservatoire des arts et métiers}

Ces cabinets, ces jardins, ainsi que les premiers musées dédiés aux sciences naturelles constitueront des références pour un effort mené parallèlement et, avec un peu de retard, dans les cabinets de physique, où, au $18^{\mathrm{e}}$ siècle, se font des expériences spectaculaires réalisées à l'aide de riches collections d'instruments scientifiques. Le cabinet de physique de Coimbra jouera un rôle pionnier dans cette démarche (fig.1). Réalisé avec le soutien des rois mécènes du Portugal, il rassemble alors une soixantaine de machines permettant de faire des démonstrations publiques et accompagne un enseignement, assuré par les professeurs (jésuites puis oratoriens) avec l'aide de machinistes, pour présenter les domaines importants de la physique (électricité, 
mécanique, optique, acoustique). Ces instruments originaux sont encore conservés à l'Université de Coimbra.

En France, l'abbé Nollet (aussi connu par ses recherches sur l'électricité et l'osmose) jouera un rôle essentiel dans cette formation par l'expérience. Ses ouvrages, tels que les Leçons de physique expérimentales éditées en 1774 , auront une grande influence sur les Grands Établissements de Science créés dans les dernières années du $18^{\mathrm{e}}$ siècle. En 1794, le Conservatoire National des Arts et Métiers (CNAM) est fondé par la Convention, dans la même mouvance que la très temporaire École Normale où l'abbé Hauÿ accompagne des cours de physique, donnés devant un millier d'élèves, de séances expérimentales [2]. L'abbé Grégoire qui pilote le projet du CNAM rassemble dans les locaux de l'abbaye de Saint-Martin-des-Champs des contenus de divers cabinets, tel que celui de mécanique de Vaucanson (avec ses automates). Des instruments techniques seront aussi rassemblés, comme le métier à tisser de Jacquard (accessoirement considéré comme le premier robot industriel à cartes perforées). Le projet du fondateur du CNAM est d'y « rassembler tous les outils et machines nouvellement inventés ou perfectionnés [...] pour éveiller la curiosité et l'intérêt ". Et il ajoute "L'enseignement, placé à côté des modèles, exige des démonstrations ", et « on évitera l'accumulation de machines inutiles ». Les instruments présentés aux élèves n'étaient donc pas considérés

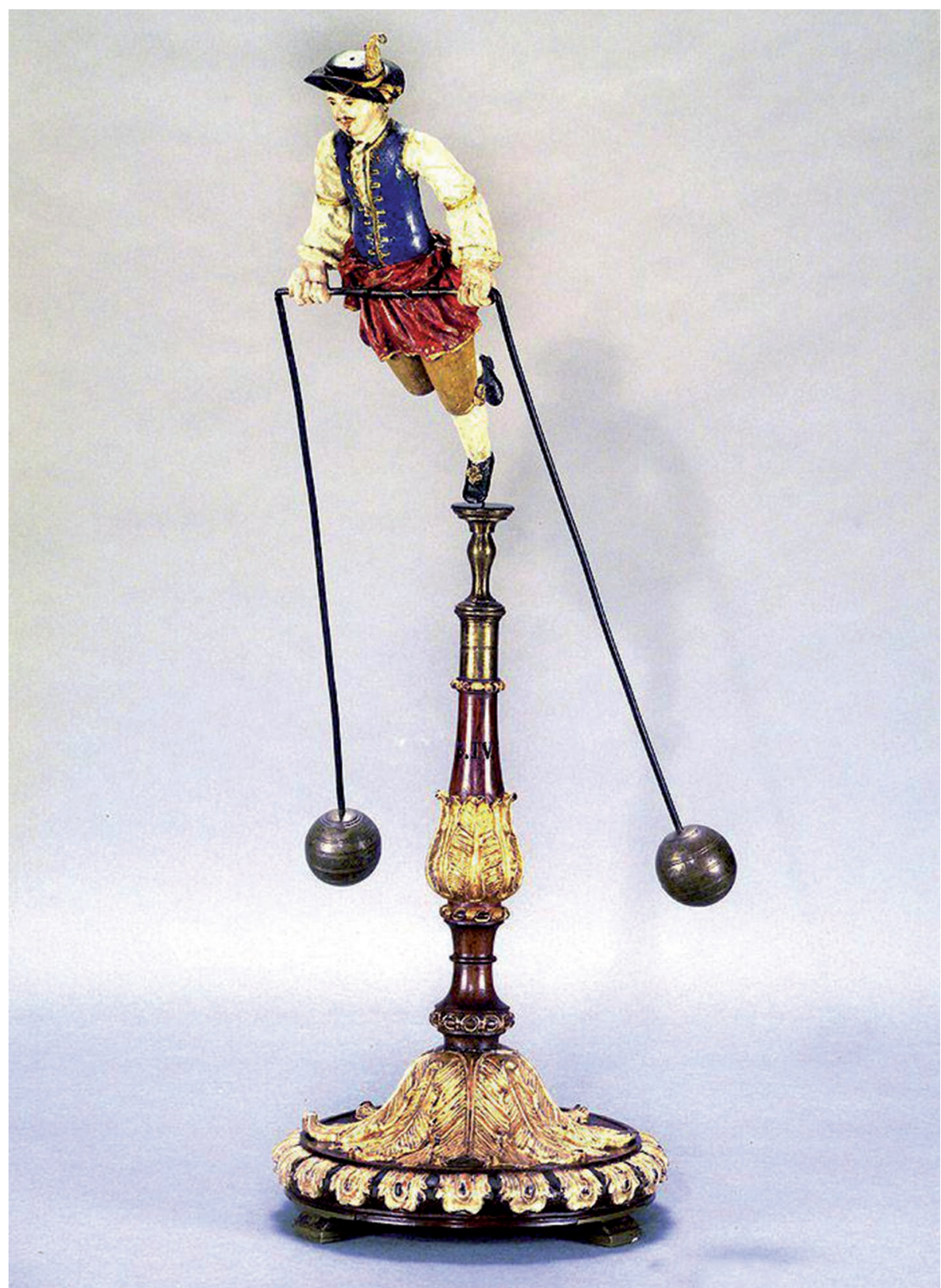

1. Équilibriste en tenue de l'époque conçu par Joaquim José dos Reis, responsable à la fin du $18^{\mathrm{e}}$ siècle de la collection des instruments de démonstration du cabinet de Physique de l'Université de Coimbra (Museu da Ciência, Universidade de Coimbra).

comme de simples objets de collections, mais étaient utilisés dans des présentations en amphithéâtre où on les déplaçait sur des chariots (dont des rails sont encore visibles sur les parquets !).

Au fur et à mesure du temps et de l'enrichissement des collections, la partie pédagogique s'estompe. En 1990, une commission ministérielle présidée par Françoise Héritier [3] conduit à proposer un ambitieux programme de rénovation pour les muséums français (une soixantaine), très souvent dans un triste état avec des accumulations d'objets de valeur rassemblés au hasard au cours du temps. Le rapport rappelle leur fonction pédagogique, distincte de celle de la collection. Cette mission sera suivie de deux " grands travaux Mitterrand ", au CNAM et au Muséum d'Histoire Naturelle de Paris. Elle conduira à la création d'un musée des Arts et Métiers, complètement recomposé aujourd'hui, et de la Galerie de l'Évolution [4]. Une approche pédagogique y est présente. Dans les deux cas, des bâtiments distincts sont dédiés aux collections. En particulier, les réserves du CNAM à Saint-Denis sont d'un grand intérêt pour l'étude des instruments de physique par des professionnels. 


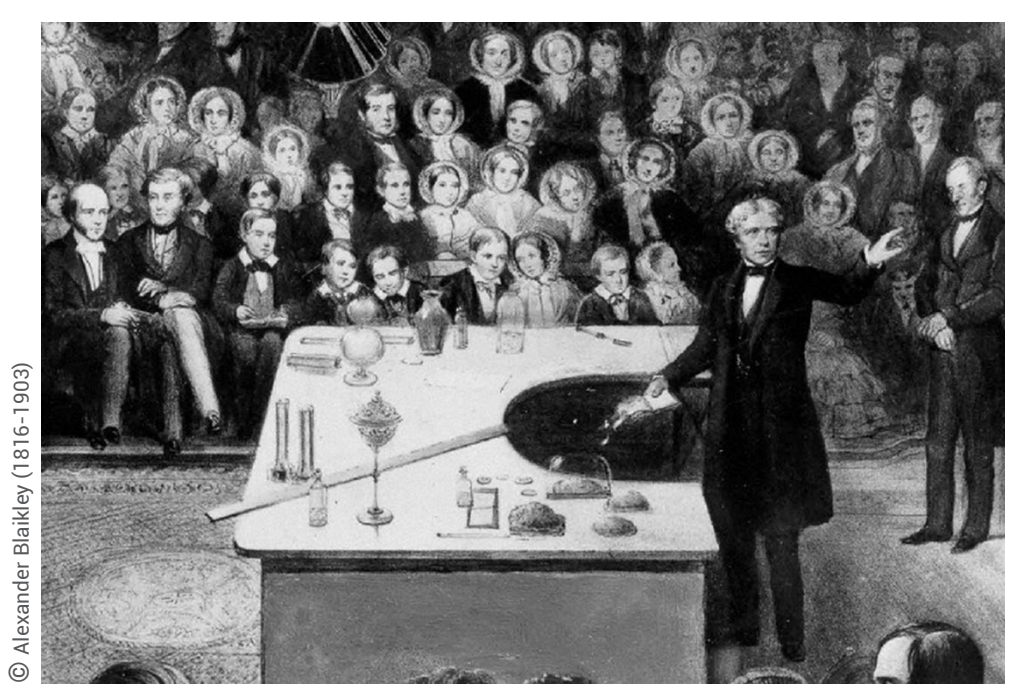

2. Michael Faraday à la Royal Institution en 1856.

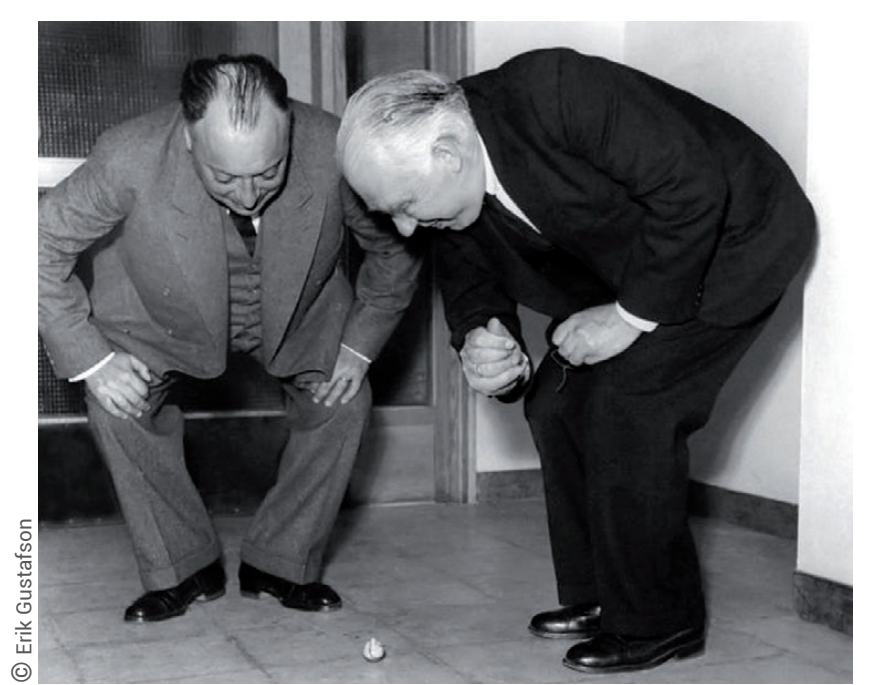

3. Wolfgang Pauli et Niels Bohr dans l'observation attentive d'une toupie tippe-top qui, quelques instants plus tard, se retournera sur la tige qui a servi à la mettre en rotation (Lund, Suède, juillet 1954) (Wikimedia commons).

》〉

\section{Le Palais de la découverte}

Un nouveau style de musée voit le jour en 1937 sous l'égide de Jean Perrin (le père du CNRS, créé dans le même temps), où l'on ne met plus en avant les objets mais les concepts. L'originalité de cette institution est de reproduire des expériences de recherche à l'aide de médiateurs ayant une formation scientifique suffisante. Ce modèle original sera appliqué dans de nombreux grands musées de science actuels, en particulier le célèbre Exploratorium de San Francisco, fondé par Franck Oppenheimer (le frère du "père " de la bombe atomique). Naturellement, ces médiateurs, en reproduisant les outils et les gestes du chercheur, sont les acteurs de leçons expérimentales ; ils proposent une démarche inductive et y introduisent une part de spectacle, mettant souvent en avant des effets contre-intuitifs. Ces présentations expérimentales au Palais de la découverte sont aussi l'objet du programme "un chercheur, une manip ", mis en place il y a une douzaine d'années, qui propose un temps d'échange direct entre un chercheur, présent pour un temps limité, et le visiteur de passage.

\section{Les leçons à la Royal Institution}

La présentation d'expériences devant le public ne constitue cependant pas une nouveauté introduite par le Palais de la découverte à Paris. La Royal Institution,
Société savante pour la diffusion de la recherche créée à Londres en 1799, est marquée par la place occupée par Michael Faraday, qui y poursuit aussi sa recherche. Il animera les "Christmas Lectures" avec des audiences de niveaux variés, et tout particulièrement des jeunes [5], dans une ambiance évoquant parfois celle d'un cirque (fig. 2). Il fera luimême près de vingt présentations, comme une série sur la flamme d'une chandelle [6]. Les plus grands scientifiques des $19^{\mathrm{e}}$ et $20^{\mathrm{e}}$ siècles (près de vingt prix Nobel) se plieront à la règle de leçons construites autour d'expériences présentées en direct de façon théâtrale et destinées à un large public. Ces conférences sont aujourd'hui diffusées par la BBC et disponibles dans leur totalité [7].

\section{Les conférences expérimentales à l'École supérieure de physique et chimie industrielles de la ville de Paris (ESPCI)}

L'expérience des leçons de la Royal Institution incitera Pierre-Gilles de Gennes à proposer à Dominique Bonnin, enseignant à l'ESPCI, un défi de taille : organiser des conférences expérimentales mensuelles sur le modèle britannique. Dominique consacrera pendant une dizaine d'années beaucoup d'énergie à la tenue de ces conférences dans le cadre de l'Espace des Sciences (qui porte désormais le nom de l'illustre directeur de l'ESPCI).L'organisation sera reprise plus tard par l'un d'entre nous, José Bico.

Les premières conférences au printemps 2003 connaissent un succès immédiat : Chimie et biologie de la beauté par Patricia Pineau de l'Oréal, puis Protection des récoltes par Vance Bergeron et David Quéré, témoignent de la diversité souhaitée des thèmes et des contributeurs. De nombreux secteurs ont été illustrés au cours de quelques 130 conférences proposées jusqu'à ce jour. Si la physique et la chimie des interfaces, la matière en grains ou la mécanique des structures sont plus représentées que la biologie, certains conférenciers talentueux n'ont pas hésité à manipuler en direct des molécules d'ADN (Vincent Croquette) ou des poissons-zèbres (Claire Wyart et Lydia Djenoune) ! Les habitués, tout comme les visiteurs occasionnels, sont unanimes : l'originalité de ces conférences tient à leur articulation autour d'expériences réalisées sur place. Cet exercice s'apparente parfois à de la haute voltige lorsqu'il s'agit par exemple de réaliser une aurore polaire sur scène (par Laurent Lamy), pour le plus grand émerveillement des spectateurs.

Nous donnons dans l'encadré 1, à titre indicatif de la diversité des thèmes, la liste des leçons présentées et prévues depuis février 2018. Si la physique occupe une place de choix dans ces exposés, on note une grande variété de thèmes avec des présentations où les sens sont sollicités. Ces présentations sont souvent faites à 
plusieurs, ce qui permet de préparer des expériences en parallèle avec l'exposé. Mais elles permettent aussi un côté plus "spectacle " avec des dialogues, se rapprochant parfois du théâtre ou du cirque, comme on avait pu le voir à la Royal Institution qui n'hésitait pas à recourir à des costumes et à une mise en scène.

Les 130 conférences données depuis leur création, toutes filmées par une équipe professionnelle, sont accessibles en ligne sur le site

$$
\text { www.espgg.org/ }
$$

Les-conferences-experimentales ainsi que, pour un grand nombre d'entre elles, sur la chaîne Canal-U, la vidéothèque numérique de l'enseignement supérieur

www.canal-u.tv/producteurs/

espci_paristech/les_conferences_ experimentales_de_1

À titre d'exemple, en 2017, les conférences de l'Espace des Sciences Pierre-Gilles de Gennes (ESPGG) diffusées sur Canal-U ont reçu plus de 40000 visites. Les enquêtes sur le public montrent trois catégories d'importance comparable : un grand public cultivé et curieux, des enseignants, et un public de chercheurs plus jeunes ne recherchant pas nécessairement sa propre discipline. Le public apprécie particulièrement les expériences en direct, ainsi que le fait que les conférenciers sont très souvent les auteurs de ces expériences.

\section{Jouons avec la physique (R. Lehoucq)}

Les conférences expérimentales font parfois appel à un délicat équipement de recherche. Au contraire, un ensemble d'expériences simples et ludiques peut servir à illustrer des phénomènes physiques très divers. Michel Courty et Édouard Kierlik, en décembre 2017, ont ainsi montré comment une caméra infrarouge permet de visualiser les échanges thermiques de la vie de tous les jours. À l'image de Niels Bohr et Wolfgang Pauli en pleine admiration devant une toupie "tippe-top " (fig. 3), une manipulation de jouets simples faite dans une conférence expérimentale en mars 2017 par Roland Lehoucq a permis d'illustrer les lois de la mécanique. Dans les encadrés 2 et 3 , nous présentons des expériences tirées de ce dernier exposé, qui mettent en avant leur côté étonnant, voire contre-intuitif, qui semble déjouer la loi universelle de chute des corps !
Les conférences données et prévues depuis février 2018 encadré 1

Lundi 12 février

Imagerie de vaisseaux rétiniens Michel Paques, Serge Meimon, Michael Atlan et Kate Grieve

Lundi 12 mars

Les maths dans les musiques actuelles Moreno Andreatta

Lundi 9 avril

La physique du tricot Samuel Poincloux et Frédéric Léchenault

Lundi 14 mai

Tempête dans un verre d'eau (savonneuse) José Bico, Étienne Reyssat et Benoît Roman

Lundi 4 juin

Oh hisse ! (fonctionnement du muscle et du système nerveux)

Caroline Cohen, Christophe Clanet et Vance Bergeron

Lundi 10 septembre

Le son de l'interaction : la musique peut-elle exprimer les relations humaines ?

Louise Goupil, Clément Canonne et Jean-Julien Aucouturier

Lundi 15 octobre

Quand deux plaques tectoniques se rencontrent : de la friction dans un vieux couple Lucile Bruhat et Jean-Arthur Olive

Lundi 12 novembre

La physique du chant Michèle Castellengo

Lundi 10 décembre

La friction granulaire ou comment bâtir sur du sable Axelle Amon et Jérôme Crassous

Tomber moins vite que la gravité

encadré 2

Un objet en chute libre tombe avec une accélération égale à celle de la pesanteur locale. Ce fait bien connu n'est en fait valable que si les frottements de l'air sont négligeables. Dans le cas contraire, l'objet atteint une vitesse limite et son accélération tend à s'annuler. Il est donc possible de tomber moins vite que la gravité. Mais dans la chute libre, notre œil ne voit guère comment la variation d'énergie potentielle se répartit entre augmentation de l'énergie cinétique et compensation de l'énergie dissipée par les frottements. Une manière de " voir » cette répartition consiste à observer la chute d'un yo-yo.

Considéré comme un des plus anciens jouets du monde avec la toupie et les osselets, le yo-yo est constitué de deux hémisphères aplatis, réunis par un axe autour duquel s'enroule une ficelle dont l'extrémité s'attache à un doigt. Le jeu consiste à lancer le yo-yo et à le ramener continûment de bas en haut.

L'état du yo-yo est spécifié par la distance verticale qu'il a parcourue depuis le lâcher et par l'angle dont il a tourné sur lui-même. Bien sûr les variations de ces deux coordonnées sont liées, car le yo-yo doit tourner pour dérouler la ficelle et se déplacer verticalement. Le problème de sa chute n'a donc qu'un seul degré de liberté. Si l'on néglige les frottements, aucune hésitation, son mouvement peut s'étudier grâce à la conservation de son énergie mécanique totale. L'énergie potentielle de pesanteur est transformée en énergie cinétique de chute et en énergie de rotation. Cette dernière différencie la chute du yo-yo de la chute libre et vient grever son énergie cinétique de chute. Le yo-yo descend donc plus lentement que s'il était en chute libre, et l'œil voit clairement que l'énergie cinétique manquante est passée dans la rotation.

Le rayon du moyeu du yo-yo varie en fonction de la hauteur de chute, car la ficelle n'est pas infiniment fine. Au début de la chute, ce rayon est grand car la ficelle est totalement enroulée : le yo-yo réel tourne donc moins vite qu'un yo-yo « idéal » dont la ficelle serait infiniment fine. La fraction d'énergie qui passe sous forme d'énergie cinétique de rotation est d'autant plus faible que la ficelle est épaisse (ou que le moment d'inertie du yo-yo est petit). Un yo-yo réel tombe ainsi plus vite que ne le ferait le yo-yo idéal. 
Tomber plus vite que la gravité semble contre-intuitif au premier abord. Pourtant, cette situation peut être mise en évidence grâce au slinky, un ressort métallique très mou proposé en 1943 par l'ingénieur en mécanique Richard T. James (fig. E1).

Pour observer une chute «libre " plus rapide que la gravité, tenons le jouet par une extrémité et laissons-le pendre sous son propre poids. La raideur du ressort étant faible, il s'allonge considérablement et l'écartement de deux spires successives diminue de haut en bas. Cela n'a rien d'étonnant car, à l'équilibre, chaque segment du ressort soutient le poids de la masse qui se trouve au-dessous de lui. Comme cette force diminue de haut en bas, l'écartement de deux spires hautes (qui est approximativement proportionnel à la force appliquée) est plus important que celui de deux spires basses.

Lâchons maintenant le slinky et observons sa chute. Même si tout va assez vite, l'œil nu perçoit que l'extrémité inférieure ne bouge pas et reste immobile tant qu'elle n'est pas rattrapée par la partie supérieure ! Le visionnage au ralenti d'une vidéo de cette chute [8] ou de photos successives prises à la manière de la chronophotographie de Marey (fig. E1) révèle plus de détails. Le ressort en chute libre présente deux parties : une zone inférieure immobile, qui conserve l'état de déformation qu'elle avait lorsque le ressort était tenu, et une zone supérieure qui tombe, où les spires sont jointives et le ressort totalement replié. Pourquoi cela?

Au moment où le slinky est lâché, son segment supérieur subit, outre son propre poids, une force dirigée vers le bas due à la tension du ressort. Il s'ensuit que l'accélération de ce segment sera supérieure à l'accélération de la pesanteur, car la force totale qu'il subit dépasse son poids. Autrement dit, ce segment tombe plus vite que s'il était en chute libre ! En revanche, une spire du bas reste immobile car encore à l'équilibre entre la tension du ressort et son poids. Après le lâcher, l'écartement des spires du haut se réduit, le ressort se raccourcit et sa tension interne au niveau de son segment supérieur diminue. La tension subie par l'extrémité haute du segment suivant diminue donc aussi, alors que la tension subie par son extrémité basse ne varie pas. Ce deuxième segment amorce alors sa chute, et ainsi de suite pour les segments suivants.

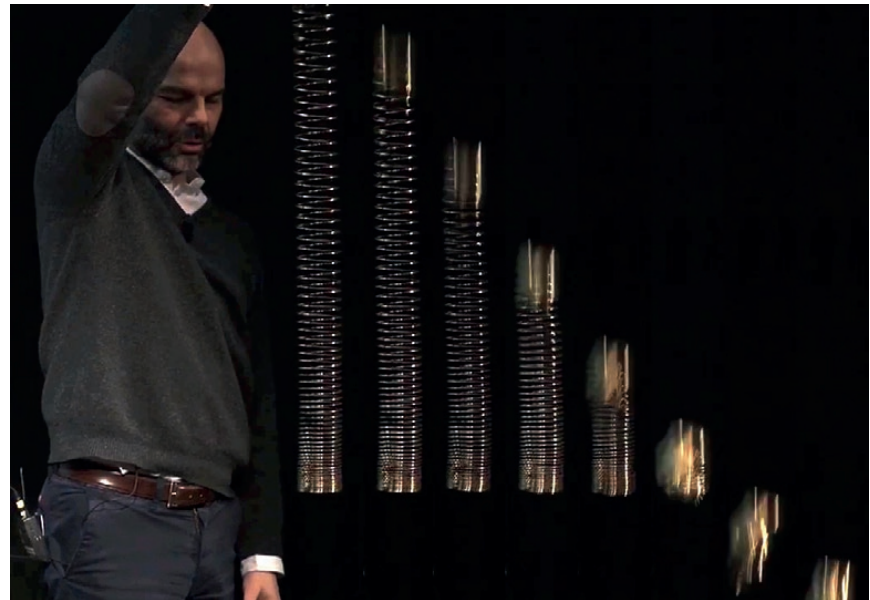

E1. Roland Lehoucq montre que lorsqu'on lâche le haut du slinky, son extrémité inférieure ne descend pas tant que le ressort n'est pas totalement comprimé. Les images successives sont séparées de 40 ms.

L'information « le slinky est lâché » n'est donc ressentie en un point du ressort que quand celui-ci est atteint par l'onde de compression des spires, qui se propage de proche en proche selon le mécanisme décrit plus haut. Comme le slinky est un ressort très mou, la vitesse de cette onde de compression est inférieure à la vitesse de chute acquise en une fraction de seconde par les spires. La chute s'amorce donc par les spires du haut qui se resserrent avant de tomber en bloc, entraînant dans leur chute les spires de la partie inférieure restées immobiles. Le centre de gravité du ressort considéré comme un tout a une accélération égale à celle de la pesanteur, heureusement ! Cependant, comme sa partie inférieure est immobile, sa partie supérieure accélère plus vite que l'accélération de la pesanteur. Quand le ressort retrouve sa longueur au repos, il tombe d'un bloc, avec l'accélération de la pesanteur, heureusement encore.

Ce phénomène est a priori présent dans tous les objets en chute libre. Mais, en général beaucoup plus rigides que le slinky, ils sont très peu déformés par leur propre poids, et les tensions internes se relâchent en une durée bien plus courte que la durée caractéristique de chute : quand un objet rigide est lâché, son bas et son haut tombent donc en bloc.

\section{Références}

$1 \cdot \mathrm{N}$. Hulin, L'enseignement secondaire scientifique en France d'un siècle à l'autre, 1802-1980, INRP (2007)

2• É. Guyon (dir.), L'École normale de I'an III. Leçons de physique, chimie et d'histoire naturelle, Éditions Rue d'Ulm (2006).

3• F. Héritier-Augé (dir.), Les musées de l'Éducation nationale : Mission d'étude et de réflexion, La Documentation française (1991).
4- Les grands travaux prévus alors pour le Palais de la découverte ne pourront se faire par suite de problèmes structuraux du Grand Palais, qui avaient été identifiés dès cette période.

5. "At my juvenile Christmas lectures, I have never found a child too young to understand intelligently what I told him; they came to me afterwards with questions who proved their capability", M. Faraday.
6• Les expériences proposées par Louis Boyer dans Feu et flammes, Belin (2006), sont un prolongement d'un tel programme.

7• www.rigb.org/christmas-lectures/Watch

8. video "How does a slinky fall ?" de la chaine Youtube Veritasium www.youtube.com/watch?v=mAA613hqqZ0 